\title{
L'urocortine : un nouveau neuropeptide anorexigène
}

Les recherches sur les effets des neuropeptides dans la régulation de la prise alimentaire ont connu récemment un développement spectaculaire dont médecine/sciences s'est fait l'écho $\left(\mathrm{m} / \mathrm{s} n^{\circ} 5\right.$, vol. $\left.12, p .625\right)[1,2]$. Parmi les peptides les plus en vogue, citons la galanine $\left(\mathrm{m} / \mathrm{s} n^{\circ} 5\right.$, vol. 12 , p. 624), le neuropeptide Y (NPY) $\left(\mathrm{m} / \mathrm{s} n^{\circ} 10\right.$, vol. $\left.12, p .1134\right)$ et la melanin-concentrating hormone $(\mathrm{MCH})$ pour leurs effets orexigènes [3-5] et le glucagon-like peptide-1 (GLP-1) $\left(\mathrm{m} / \mathrm{s} n^{\circ} 3\right.$, vol. $12, p$. 392) et la cholécystokinine (CCK) pour leur effet modérateur sur la prise de nourriture $\left(\mathrm{m} / \mathrm{s} n^{\circ} 8 / 9\right.$, vol. $12, p$. 972) [6, 7]. L'urocortine, un peptide apparenté au corticotropin-releasing factor (CRF) récemment découvert dans le cerveau du rat $\left(m / s n^{\circ} 2\right.$, vol. $12, p$. 249), vient grossir les rangs des neuropeptides susceptibles de régler le comportement alimentaire.

Depuis la caractérisation du CRF en 1981 [8], il était communément admis qu'il n'existait qu'une seule forme de ce neuropeptide dans le cerveau des vertébrés. En revanche, deux peptides présentant de fortes similitudes de séquence avec le CRF, la sauvagine et l'urotensine I, avaient été identifiés respectivement à partir de la peau de la grenouille [9] et de l'urophyse des poissons [10]. L'urotensine II, un autre peptide initialement caractérisé dans l'urophyse [11], a été ultérieurement identifié dans le cerveau de la grenouille [12, 13], suggérant que les urotensines ou des peptides apparentés pouvaient également être synthétisés dans le cerveau des mammifères. Partant de cette hypothèse, l'équipe de J. Rivier et W. Vale (Salk Institute, La Jolla, CA, USA) a caractérisé un nouveau gies avec l'urotensine I et avec le CRF et qui, de ce fait, a été désigné sous le terme d'urocortine [14].

Il est bien connu que le CRF, outre son action neuro-endocrinienne stimulatrice de l'axe corticotrope, induit diverses réactions comportementales qui miment certaines réponses au stress. En particulier, l'administration centrale de CRF diminue l'activité locomotrice, induit des manifestations d'anxiété et réduit la prise alimentaire. L'équipe de G.F. Koob (Scripps Research Institute, La Jolla, CA, USA), en collaboration avec J. Rivier et W. Vale, vient de montrer que l'injection intracérébroventriculaire d'urocortine provoque une anorexie plus intense que celle induite par le CRF [15]. En revanche, les effets anxiogènes de l'urocortine sont nettement moindres que ceux du CRF. L'urocortine diminue à la fois la prise de nourriture et la prise de boisson chez les animaux à jeun comme chez les animaux correctement nourris. Les effets de l'urocortine sur la consommation alimentaire sont du même ordre que ceux induits par la D-fenfluramine, un puissant agent anorexigène.

Deux types de récepteurs du CRF ont été caractérisés à ce jour $\left(m / s n^{\circ} 8\right.$, vol. 11, p. 1185). Plusieurs observations suggèrent que les effets anorexigènes de l'urocortine sont relayés par les récepteurs de type 2 (CRF-R2): (1) l'urocortine présente une plus grande affinité pour le CRF-R2 que pour le CRF-R1; (2) plusieurs régions du cerveau impliquées dans le contrôle de la prise alimentaire ou la régulation des fonctions digestives possèdent à la fois des terminaisons nerveuses à urocortine et des récepteurs de type CRF-R2. Malheureusement, les antagonistes des récepteurs du CRF disponibles à l'heure actuelle ne permettent pas de discriminer ces deux types de récepteurs. Il faudra donc attendre que soit réalisée l'invalidation des gènes codant pour le précurseur de l'urocortine ou pour le récepteur de type CRF-R2 pour savoir si ce nouveau neuropeptide joue un rôle physiologique dans la régulation du comportement alimentaire.

H.V.

1. Castan I, Valet P, Lafontan M. Le récepteur du PYY : un élément important du système antilipolytique de l'adipocyte. Med Sci 1994; 10: 196-201.

2. Guerre-Millo M, Saladin R, Staels B, Auwerx J. Les facteurs régulateurs du gène ob. Med Sci 1996 ; $12: 383-5$.

3. Akabayashi A, Doenig JI, Watanabe Y, Alexander JT, Leibowitz SF. Galanin-containing neurons in the paraventricular nucleus: a neurochemical marker for fat ingestion and body weight gain. Proc Natl Acad Sci USA 1994; 91 : 10375-9.

4. Gerald C, Walker MW, Criscione L, Gustafson EL, Batzl-Hartmann C, Smith KE, Vaysse P, Durkin MM, Laz TM, Linemeyer DL, Schaffhauser AO, Whitebread S, Hofbauer KG, Taber RI, Branchek TA, Weinshank RL. A receptor subtype involved in neuropeptide Y-induced food intake. Nature 1996; 382 : 168-71.

5. Qu D, Ludwig DS, Gammeltoft S, Piper M, Pelley-mounter MA, Cullen MJ, Foulds Mathes W, Przypek J, Kanarek R, Maratos-Flier E. A role for melanin-concentrating hormone in the central regulation of feeding behaviour. Nature 1996; 380: 243-6.

6. Turton MD, O'Shea D, Gunn I, Beak SA, Edwards CMB, Meeran K, Choi SJ, Taylor GM, Heath MM, Lambert PD, Wilding JPH, Smith DM, Ghatei MA, Herbert J, Bloom SR. A role of glucagon-like peptide-1 in the central regulation of feeding. Nature 1996; 379: 69-72.

7. Griesbacher T, Leighton GE, Hill RG, Hughes $\mathrm{J}$. Reduction of food intake by central administration of cholecystokinin octapeptide in the rat is dependent upon inhibition of brain peptidases. Br J Phamacol 1989; 96: 236-42.

8. Vale W, Spiess J, Rivier C, Rivier J. Characterization of a 41 -residue ovine hypothalamic peptide that stimulates secretion of corticotropin and $\beta$ endorphin. Science 1981 ; 213: 1394-7. 
9. Montecucchi PC, Henschen A. Amino acid composition and sequence analysis of sauvagine, a new active peptide from the skin of Phyllomedusa sauvagii. Int J Pept Prot Res 1981 ; 18: 113-20.

10. Lederis K, Letter A, McMaster D, Moore G, Schlesinger D. Complete amino acid sequence of urotensin I, a hypotensive and corticotropin-releasing neuropeptide from Catostomus. Science 1982; 218: 162-4.

I1. Pearson D, Shively JE, Clark BR, Geschwind II, Barkley M, Nishioka RS, Bern HA. Urotensin II : a somatostatin-like peptide in the caudal neurosecretory system of fishes. Proc Natl Acac Sci USA $1980 ; 77: 5021-4$.

12. Conlon JM, O'Harte F, Smith DD, Tonon MC, Vaudry $\mathrm{H}$. Isolation and primary structure of urotensin II from the brain of a tetrapod, the frog Rana ridibunda. Biochem Biophys Res Commun 1992; 188: 578-83.

13. Chartrel N, Conlon JM, Collin F, Braun B, Waugh D, Vallarino M, Lahrichi SL, Rivier JE, Vaudry H. Urotensin II in the central nervous system of the frog Rana ridibuna: immunohistochemical localization and biochemical characterization. J Comp Neurol 1996; 364: 324-39.

14. Vaughan J, Donaldson C, Bittencourt J, Perrin $\mathrm{MH}$, Lewis K, Sutton S, Chan R, Turnbull AV, Lovejoy D, Rivier C, Rivier J, Sawchenko PE, Vale W. Urocortin, a mammalian neuropeptide related to fish urotensin I and to corticotropin-releasing factor. Nature 1995; 378: 287-92.

15. Spina M, Merlo-Pich E, Chan RKW, Basso AM, Rivier J, Vale W, Koob GF. Appetite-suppressive eff ects of urocortin, a CRF-related neuropeptide. Science 1996 ; 273: 1561-4.

Société française de biologie du développement (SFBD)

Membre de l'European Developmental Biology Organization (EDBO)

Colloque annuel 1997:

Développement et évolution

Dourdan (France) 29-31 mai 1997

Thèmes évoqués: Évolution des systèmes géniques et morphogenèse. Développement et voies de signalisation. Origine des lignages cellulaires. Morphogenèse des végétaux. Développement et stratégies adaptatives. Développement en apesanteur.

Conférenciers invités: André Adoutte; Michael Akam; Jo van den Biggelaar; Jean-Claude Boucault; Herman Denis; Denis Duboule; Claudie LamourIsnard; Manuel Mark; Nipam Patel; Armand de Ricqlès; Pat Simpson et la spationaute Claudie André-Deshays (Centre National d'Études Spatiales). Comité scientifique: André Adoutte; Pierre Chambon; Jean Deutsch; Denis Duboule; AnneMarie Duprat; François Jacob; Axel Kahn; Nicole Le Douarin; Jean-Antoine Lepesant; Armand de Rouarin; Jean-Antoine
Ricqlès; Maurice Wegnez.

Comité d'organisation: Yannick Andéol; Christophe Chanoine; Jean Foucrier; Jacqueline Géraudie; JeanPierre Muller; May Penrad-Mobayed; Benoit Robert Maurice Wegnez.

Date limite d'inscription et d'envoi des résumés: 15 mars 1997

Contacts: Pr Maurice Wegnez, Colloque SFBD 97 Laboratoire d'Embryologie Moléculaire, Universite Parid XI, Bât. 445, 91405 Orsay. Tél: 0169157287 ; Fax: 01691568 02; e-mail : wegnez@popu.u-psud.fr.

\section{APPEL D'OFFRES}

PRIX

\section{INSERM/ACADEMIE DES SCIENCES}

1997

\section{Un prix d'un montant total de $200000 \mathrm{FF}$, destiné à apporter} un soutien important aux travaux de recherche d'une équipe signataire

d'une Note de grande portée scientifique publiée

dans les Comptes Rendus de l'Académie des Sciences, dans les domaines de la biologie fondamentale, des sciences biomédicales et de la santé, sera attribué en 1997.

Les notes concourant pour ce Prix auront été publiées dans les Comptes Rendus de l'Académie des Sciences des années 1994, 1995, 1996, Série III (Sciences de la Vie). Elles doivent faire l'objet d'une proposition par une personnalité scientifique ou par les auteurs eux-mêmes. L'évaluation des dossiers sera effectuée

par le Conseil scientifique de l'INSERM et l'Académie des Sciences.

Les dossiers de candidature, disponibles à l'INSERM et au secrétariat de l'Académie des Sciences, doivent être adressés à l'INSERM.

Date limite de dépôt des candidatures : 28 mars 1997. La décision d'attribution du Prix sera communiquée fin juillet 1997.
Institut National de la Santé et de la Recherche Médicale Département de l'Information et de la Communication 101, rue de Tolbiac 75654 Paris cedex 13

Tél. : 01.44.23.60.71
Académie des Sciences

Secrétariat

23, quai de Conti - 75006 Parịs

Tél. : 01.44.41.43.83

\section{ACADEMIE DES SCIENCES}

INSTITUT NATIONAL DE LA SANTE ET DE LA RECHERCHE MEDICALE 